\title{
Analysis of preoperative and postoperative quality of life, sexual function, and sleep in patients with endometriosis: A prospective cohort study
}

Belfin Nur Arici Halici

Bezmialem University Faculty of Medicine https://orcid.org/0000-0002-8822-4740

Fatih Aktoz

VKV American Hospital

Meric Kabakci

University of Health Sciences https://orcid.org/0000-0002-6956-6753

Gurkan Kiran

Bezmialem University Faculty of Medicine https://orcid.org/0000-0002-6300-328X

Pinar Özcan ( $\nabla$ drpinarozcan@hotmail.com )

Bezmialem Vakif Universitesi https://orcid.org/0000-0002-0306-6422

\section{Research Article}

Keywords: endometriosis, quality of life, sexual function, quality of sleep

Posted Date: November 10th, 2021

DOI: https://doi.org/10.21203/rs.3.rs-1048253/v1

License: (9) This work is licensed under a Creative Commons Attribution 4.0 International License.

Read Full License

Version of Record: A version of this preprint was published at Archives of Gynecology and Obstetrics on April 22nd, 2022. See the published version at https://doi.org/10.1007/s00404-022-06562-9. 


\section{Abstract \\ Purpose}

Endometriosis affects the quality of life, sleep, and sexual life of patients due to pain. This study compared the scores of endometriosis patients in these three areas before and after surgery.

\section{Methods}

Patients between the ages of 18-60 with a prediagnosis of endometriosis were enrolled. Postoperative histopathological diagnosis of endometriosis was confirmed in all patients. This study included 56 patients who completed pre- and postoperative (three months) evaluation of quality scale questionnaires: a visual analog scale for pelvic pain, the Pittsburgh Sleep Quality Index, Morningness-Eveningness Questionnaire, Endometriosis Health Profile-30 Questionnaire, and Female Sexual Function Index were administered prior to and three months after each patient's surgery.

\section{Results}

Among the 56 female patients included in this study, statistically significant improvement statistically significant improvement was observed in pain scores, quality of life, sexual function, and sleep of all patients regardless of endometriosis stage.

\section{Conclusion}

Endometriosis is a disease that progresses, with increasing pain scores; it has negative effects on the quality of life, sexual function, and sleep of patients. Surgical or medical treatment can be performed considering the complaints and fertility status of the patients.

\section{Introduction}

Endometriosis is a gynecological disease characterized by the existence of endometrial glands and tissue outside the uterus. Since the diagnosis can be made only by histopathological examination, the prevalence of the disease could not be determined clearly. While endometriosis was detected in $5-60 \%$ of women with pelvic pain and unexplained infertility, the condition was also found in $1-7 \%$ of asymptomatic patients [1]. In another study, $50-70 \%$ of adolescents examined for severe dysmenorrhea were diagnosed with endometriosis [2]. In a study conducted on women who underwent laparoscopic intervention in ten different countries on five different continents, it was determined that endometriosis was a prevalent chronic disease and its incidence was $35-100 \%$ in symptomatic patients [3]. Most patients with endometriosis have complaints of pelvic pain, dysmenorrhea, and dyspareunia. This pain can be explained by the direct and indirect effects of local bleeding in endometriotic implants, 
inflammatory cytokine effects in the peritoneal cavity, pelvic floor nerve irritation, or direct infiltration. In addition, patients' quality of life, sexual life, and sleep are adversely affected due to pain. These three components are closely related to each other and to the overall health status of the patient.

Quality of life is a complex concept that includes an individual's physical, mental, sociocultural, and educational conditions. In the literature, it was reported that there was a strong relationship between endometriosis-related symptoms and poor quality of life [4]. Patients with endometriosis reported reduced quality of life, increased stress levels, and more depressive symptoms compared to patients with idiopathic infertility [5]. Sexuality is a multifaceted identity that affects physical health, psychological well-being, femininity, and relationships. Sexual function is affected by medical conditions and physical, psychological, and sociocultural factors. Given the physiological, psychological, and social effects of endometriosis, it is unsurprising that it negatively affects women's sexual lives [6]. Women with chronic diseases may have worse sexual function due to pain [6].

Sleep plays an important role in vital functions. The circadian rhythm is a biological process that prepares the organism for predictable changes in the environment that occur across a 24-hour period. Recognition of individual variations in the circadian rhythm have led to the development of the concept of chronotypes. A person's chronotype affects the level and intensity of their cognitive and physical activity during throughout the day. There are three chronotypes: Morning-type, evening type, and intermediate type. Morning-type people prefer to be active early in the day, while evening-type people are active late in the day. Most of the population is of the intermediate type. In the literature, it was shown that morning people had a more stable circadian rhythm and a healthier mental and physical life compared to the evening types [7]. Also, optimal sleep levels have been found to be associated with a reduced risk of death from all causes and an increased quality of life [8]. Therefore, a high quality of sleep is essential for the maintenance of social, emotional, and physical well-being, as well as for the process of healing injuries and recovering from diseases.

Limited data exist to evaluate pre- and postoperative quality of life, sexual function and sleep quality of women diagnosed with endometriosis while interaction between endometriosis and quality of life, sexual life and sleep quality is an important clinical condition. It is hypothesized that there could be strong relationship between these conditions and endometriosis and there would be a tendency for the improvement of these conditions after surgery. For this reason, we aim to evaluate the impact of endometriosis on the quality of life, sexual function and sleep quality and compare the pre- and postoperatively alteration of all conditions to determine the effect of endometriosis surgery via validated questionnaires as well.

\section{Materials And Methods}

A total of 56 women attending the Department of Obstetrics and Gynecology

of Bezmialem University Hospital between 2020 and 2021 were included. This prospective cohort study protocol was approved by the Ethical Committee of the Medical Faculty of Bezmialem University. Written 
informed consent was obtained from all patients

Inclusion criteria were women between 18 and 60 years old with a preliminary diagnosis of endometriosis, willingness to participate in the study and underwent surgery in our clinic. All diagnosis was postoperatively histopathologically confirmed as endometriosis. Exclusion criteria were patients with a previously known mental, neurologic disorders or sleep disorders who received treatment, nonwillingness for participation, patients who did not have an operation indication but received medical treatment, patients who had not had sexual intercourse before, patients under the age of 18 and over the age of 60 , women with night shift work and patients who used drugs that affect the sleep-wake cycle.

The data collected included demographic characteristics of the patients (age, alcohol consumption, smoking status, chronic disease history, gravida, parity, education level, occupation, and body mass index); stage of endometriosis according to the American Society for Reproductive Medicine (ASRM) staging system; Morningness-Eveningness Questionnaire (MEQ) groups and score; Pittsburgh Sleep Quality Index (PSQI) groups and score; visual analog scale (VAS); the Endometriosis Health Profile-30 Questionnaire (EHP-30), and the Female Sexual Function Index (FSFI). The evaluation of scale questionnaires for each patient were performed twice as prior to operation and three months after operation.

\section{Visual analog scale (VAS)}

VAS is a tool for indicating values that are difficult to express numerically by selecting them over a spectrum. One end of a $10 \mathrm{~cm}$ line is labeled as "no pain", and the other end as "the worst pain imaginable." The patient indicates the extent of the pain they feel by marking a point on this spectrum. This point is then analyzed numerically. VASs are widely used for pain measurements.

\section{Pittsburgh sleep quality index (PSQI)}

PSQI, which is widely used in relation to sleep quality, has been found useful in the sleep disorder research approach [9]. This index contains a total of 24 questions, 19 of which are answered by the individual and five that are answered by the spouse of the individual or a person with whom they live. When calculating the score, questions answered by people other than the individual are not included in the calculation. The questions are about sleep duration, sleep latency, frequency, and severity of special sleeping problems. These 19 scored items are grouped into seven modules: subjective sleep quality, sleep latency, sleep duration, sleep efficiency, sleep disturbance, use of sleep medication, and daytime dysfunction. Some are created from a single item, while others are created by grouping several items. Each module is evaluated with a score between $0-3$. The sum of these seven component scores gives an overall index score, between 0-21. A total PSQI score of five or more shows poor quality of sleep.

\section{Morningness-eveningness questionnaire (MEQ)}

MEQ is the scale used to determine chronotype. The questionnaire consists of 19 questions about the physical and psychological performance of individuals across a 24-hour period of time and their 
preferred times for various activities. Scoring varies between 16-86; scores between 16-41 indicate evening type, scores between 42-58 indicate intermediate type, and scores between 59-86 indicate morning type.

\section{Endometriosis Health Profile-30 Questionnaire (EHP-30)}

EHP-30 measures endometriosis-related life status on 30 disease-compatible questionnaires and five scales (pain, control and weakness, emotional well-being, social support, and self-image). The questions evaluate sexual intercourse, work, relationship with children, feelings about the medical profession, treatment, and infertility.

\section{Female Sexual Function Index (FSFI)}

FSFI is frequently used in sexual dysfunction studies. FSFI is a 19-item questionnaire. It covers six main topics-sexual desire, arousal, lubrication, orgasm, satisfaction, and pain. Scoring ranges from 1-5 for questions $1,2,15,16$, and $0-5$ for other questions. The total score of each area is multiplied by a certain ratio. This ratio is 0.6 for desire; 0.4 for orgasm, satisfaction, and pain; and 0.3 for arousal and lubrication. Overall, higher scores indicate a better sexual life.

\section{Statistical analysis}

Statistical analyses were performed using the McNemar test, McNemar Bowker test, Wilcoxon test, and the Kruskall Wallis test, and for pairwise comparisons the Dunn-Bonferroni post-hoc test. The significance level was taken as $a=0.05$. Mean \pm standard deviation was given for continuous variables. Percentage and number values were noted for categorical variables. The enter method was used for binary logistic regression analysis. Analyses were made with SPSS v. 25 (SPSS Inc., Chicago, IL, USA).

\section{Results}

Full demographic and clinical characteristics of the patients are summarized in Table 1. The mean age of 56 women included in the study was $36.82 \pm 7.77$ (ranging from 22-54). The mean age at first onset of pelvic pain was $30.86 \pm 7.93$. According to the ASRM staging system for endometriosis, $35.7 \%(n=20)$ of the patients were in stage $1,28.6 \%(n=16)$ were stage $2,14.3 \%(n=8)$ were in stage 3 , and $21.4 \%(n=$ 12) were in stage 4. 
Table 1

The demographic and clinical characteristics of the patients

\begin{tabular}{|ll|}
\hline Age (year) & $36,82 \pm 7.77$ \\
\hline First onset of pelvic pain (year) & $30,86 \pm 7,93$ \\
\hline Gravidity & $1,39 \pm 1,47$ \\
\hline Parity & $1,18 \pm 1,09$ \\
\hline Body Mass Index $\left(\mathrm{kg} / \mathrm{m}^{2}\right)$ & $25,29 \pm 4,86$ \\
Stage of disease & $20(\% 35,7)$ \\
Stage 1 & $16(\% 28,6)$ \\
Stage 2 & $8(\% 14,3)$ \\
Stage 3 & $12(\% 21,4)$ \\
Stage 4 & $7(\% 12,5)$ \\
Alcohol comsumption & $20(\% 35,7)$ \\
Smoking & $17(\% 30,4)$ \\
Chronic disease & \\
\hline Mean \pm standard deviation & \\
\hline
\end{tabular}

An overview of our findings are presented below; the full pelvic pain VAS, PSQI, MEQ, EHP-30, and FSFI scores of the patients are shown in Table 2. The mean preoperative VAS scores for dysmenorrhea, dyspareunia, and chronic pelvic pain (CPP) were $7.79 \pm 1.26(2.73 \pm 1.49), 6.66 \pm 2.48$ (3.27 \pm 1.40$)$, and $6.98 \pm 1.73(3.04 \pm 1.26)$, respectively. There was a large and statistically significant decrease in VAS scores in the postoperative period $(p<0.001)$. 
Table 2

Comparison of preoperative and postoperative groups

\begin{tabular}{|c|c|c|c|}
\hline Parameters & Preoperatively & Postoperatively & p-value \\
\hline VAS score & $7,79 \pm 1,26$ ** & $2,73 \pm 1,49 * \star$ & $<0,001^{*}$ \\
\hline \multicolumn{4}{|l|}{ Dysmenorrhea } \\
\hline Dyspareunia & $6,66 \pm 2,48^{\star \star}$ & $3,27 \pm 1,40 * \star$ & $<0,001^{*}$ \\
\hline Chronic Pelvic Pain & $6,98 \pm 1,73^{\star \star}$ & $3,04 \pm 1,26$ ** & $<0,001^{*}$ \\
\hline PSQI score & $6,54 \pm 3,15^{\star \star}$ & $3,18 \pm 1,08 * \star$ & $<0,001^{*}$ \\
\hline \multicolumn{4}{|l|}{ PSQI groups } \\
\hline$<5$ & $13(\% 23,2)^{\wedge}$ & $53(\% 94,6)^{\wedge}$ & \\
\hline$>5$ & $43(\% 76,8)^{\wedge}$ & $3(\% 5,4) \wedge$ & \\
\hline \multicolumn{4}{|l|}{ PSQI subgroups } \\
\hline Subjective sleep quality & $1,34 \pm 0,85^{\star \star}$ & $0,77 \pm 0,60$ ** & $<0,001^{*}$ \\
\hline Sleep latency & $1,64 \pm 0,98 * \star$ & $0,89 \pm 0,59 * \star$ & $<0,001^{*}$ \\
\hline Sleep duration & $0,54 \pm 0,66 * *$ & $0,30 \pm 0,46$ ** & $<0,001^{*}$ \\
\hline Sleep efficiency & $0,48 \pm 0,71$ ** & $0,21 \pm 0,41$ ** & $<0,001^{*}$ \\
\hline Sleep disturbances & $1,48 \pm 0,71$ ** & $0,75 \pm 0,43^{\star *}$ & $<0,001^{*}$ \\
\hline Use of sleep medication & $0,18 \pm 0,47 \star \star$ & $0,04 \pm 0,18 * *$ & $<0,001^{*}$ \\
\hline Daytime dysfunction & $0,80 \pm 0,92^{\star \star}$ & $0,18 \pm 0,43^{\star *}$ & $<0,001^{*}$ \\
\hline MEQ score & $48,95 \pm 10,58$ ** & $52,98 \pm 9,0 * \star$ & $<0,001 *$ \\
\hline EHP-30 score & $52,66 \pm 19,1^{\star *}$ & $29,41 \pm 11,37$ ** & $<0,001 *$ \\
\hline FSFI score & $11,56 \pm 6,17 \star \star$ & $16,57 \pm 6,13^{\star \star}$ & $<0,001 *$ \\
\hline \multicolumn{4}{|l|}{${ }^{*} p<0,05$} \\
\hline \multicolumn{4}{|c|}{ ** Mean \pm standard deviation } \\
\hline Number and percentage & & & \\
\hline
\end{tabular}

The mean preoperative PSQI score was $6.54 \pm 3.15$, and the postoperative score dropped to $3.18 \pm 1.08$. While the preoperative good sleep quality rate was $23.2 \%(n=13)$ and the poor sleep quality rate was $76.8 \%(n=43)$, the postoperative good sleep quality rate increased dramatically, to $94.6 \%(n=53)$, and the poor sleep quality rate decreased to just $5.4 \%(n=3)$. Of those who had poor sleep quality prior to their operations, $75.5 \%(n=40)$ reported good sleep quality three months afterwards. When pre- and postoperative sleep quality was compared, this substantial change in the results was found to be 
statistically significant $(p<0.001)$. When the PSQI subgroups were examined, the mean values of subjective sleep quality, sleep latency, sleep duration, sleep efficiency, sleep disturbance, use of sleep medication, and daytime dysfunction in the preoperative vs postoperative period were $1.34 \pm 0.85$ vs 0.77 $\pm 0.60,1.64 \pm 0.98$ vs $0.89 \pm 0.59,0.54 \pm 0.66$ vs $0.30 \pm 0.46,0.48 \pm 0.71$ vs $0.21 \pm 0.41,1.48 \pm 0.71$ vs $0.75 \pm 0.43,0.18 \pm 0.47$ vs $0.04 \pm 0.18$, and $0.80 \pm 0.92$ vs $0.18 \pm 0.43$, respectively, and there was a statistically significant differences between pre and postoperative period $(p<0.01)$.

The mean MEQ scores were $48.95 \pm 10.58$ preoperatively and $52.98 \pm 9.0$ postoperatively; this change was statistically significant $(p<0.001)$. The proportion of patients who displayed morning type in both the pre- and postoperative periods was $25 \%(n=14)$. Of 18 patients who had evening type in the preoperative period, $5(27.8 \%)$ were detected as morning type, 4 (22.2\%) as evening type and 9 (50\%) as intermediate type in the postoperative period.

While the mean EHP-30 score was $52.66 \pm 19.1$ preoperatively, it decreased to just $29.41 \pm 11.37$ in the postoperative period. This represented a statistically significant improvement in patients' quality of life in the postoperative period $(p<0.001)$. The mean FSFI scores increased by 5.01 , from a preoperative 11.56 \pm 6.17 to $16.57 \pm 6.13$ postoperatively. According to these results, patients experienced statistically significant postoperative improvement in sexual function $(p<0.001)$.

There were no statistically significant correlations between disease stage and postoperative VAS, PSQI, MEQ, EHP-30, and FSFI scores ( $p>0.05)$.

\section{Discussion}

Endometriosis can cause complaints such as pelvic pain, dysmenorrhea, dyspareunia, and infertility. These complaints negatively affect patients' quality of life, sexual function, and sleep, among other aspects of life. While there have been many studies showing that endometriosis causes these complaints, there have been few investigating the effect of treatment on these complaints. Although the few studies that do exist have variously shown treatment to have both positive and negative effects on pelvic pain, dysmenorrhea, dyspareunia, and infertility complaints, the effects on quality of life, sexual function, and sleep, which are directly related to patients' lives, have not been adequately investigated. In addition, although there are a limited number of studies examining the pre- and postoperative quality of life and sexual function in patients with endometriosis, only preoperative evaluations were investigated in studies on sleep quality.

Many factors may play a role in the formation of pain. Women with endometriosis have high amounts of nerve growth factor in their peritoneal fluid. This promotes neurogenesis, which changes the ratio of sympathetic and sensory nerve fibers in endometriotic tissue and increases nerve density. At the same time, cytokines, prostaglandins, and inflammatory cells activate nerve fibers. Compression of nerve fibers within endometriotic implants also causes considerable pain, as does central hypersensitivity. Due to endometriosis-induced neuroplastic changes in the descending pathways that regulate pain perception, patients become more sensitive to pain stimuli. Patients suffer from pain in endometriosis as a result of 
insufficiency in descending inhibition mechanisms. CPP is defined as pelvic pain lasting at least 6 months. Some patients have underlying factors such as endometriosis, adenomyosis, or pelvic inflammatory disease. However, it can also be idiopathic. CPP usually occurs after an acute pain attack, even when the initial stimulus disappears [10].

Meanwhile, severity of endometriosis may not be related to the level of pain although most of the studies generally examined patients with stage 4 endometriosis [11]. There is no evidence of increased pain in advanced stages of endometriosis. Furthermore, there may be severe pain even if there is a small focus, or the pain may be very mild even if the bladder, bowel, and ureter are infiltrated [12]. However, when patients with deep infiltrative and superficial endometriosis were compared, surgical intervention was shown to reduce pain more in the former [13].

\section{Endometriosis and Quality of Life}

Endometriosis has negative effects on quality of life due to symptoms such as CPP, dysmenorrhea, and dyspareunia, especially in the reproductive years. In the pathophysiology of endometriosis-associated pain, signaling pathways and inflammatory and hormonal changes are responsible for many of these symptoms. The symptom that most affects quality of life for endometriosis patients is pain. Painful symptoms reduce physical, mental, and social welfare and have a negative impact on work performance as well as quality of life. In one study, researchers found that fully $40 \%$ of endometriosis patients could not be promoted at work due to their symptoms and resulting loss of customers [14].

CPP causes productivity problems, relationship difficulties, social dissatisfaction, and psychological morbidity in patients $[15,16]$. This may lead to social isolation, and subsequently deterioration of social relations. All this shows that endometriosis can significantly impair quality of life by causing psychological disorders such as anxiety and depression. It was shown that pain and depression improved with treatment of endometriosis, and thus, patients' quality of life increased [17]. One of the most common causes of dysmenorrhea is endometriosis. Increased endometrial prostaglandin production is mainly responsible for this mechanism. Rush et al. reported that the quality of life of patients with dysmenorrhea was adversely affected [18]. Depending on the severity of the pain in dysmenorrhea, it may severely impair the quality of life for some patients by negatively affecting their mental health and social lives [19].

There are pre- and postoperative comparative studies evaluating the negative effects of endometriosisrelated symptoms on quality of life and improvement after surgery. Parra et al. showed that half of patients with deep infiltrative endometriosis and rectosigmoid invasion who underwent laparoscopic surgery had reduced pelvic pain, increased daily activity performance, and had better work performance [20]. In a retrospective study, health care providers observed that dysmenorrhea, dyspareunia, and CPP complaints decreased by the three-month postoperative evaluation in patients with deep infiltrative endometriosis [21]. Likewise, in another study comparing definitive surgery and uterine-sparing laparoscopic excision in the treatment of endometriosis, it was observed that there was an improvement 
in the quality of life in both groups postoperatively [22]. In a randomized controlled study, a decrease in pain scores and an increase in quality of life were observed during patients' 6 - and 12-month follow-ups [23].

Our results revealed that regardless of stage of endometriosis, all patients was provided with definitive surgery contributed to the improvement of quality of life in the postoperative period. With the results we obtained in our study, it can be said that the operation is beneficial for endometriosis patients in all stages in terms of quality of life.

\section{Endometriosis and Quality of Sexual Life}

In general, patients with endometriosis are considered to have deep dyspareunia. However, in most studies, any pain experienced during sexual intercourse was defined as dyspareunia. In the presence of dyspareunia, pain results in sexual dysfunction. Fear of this pain has negative effects on sexual desire, arousal, and lubrication. In addition, central sensitization causes hyperalgesia, allodynia, and worsening of pain perception. Factors such as anxiety, guilt after coitus, and disappointment also negatively affect sexual motivation by creating emotional stress [24]. The frequency of orgasm, sexual pleasure, and satisfaction was found to be lower in patients experiencing pain [25]. Another cause of sexual dysfunction is depression due to dyspareunia and the effect of anxiety. Due to this, feelings of guilt due to not being able to have intercourse, deterioration in ideal body image, and an increase in sexual dysfunction were observed in patients [26].

A prospective study in patients diagnosed with endometriosis revealed a relationship between dyspareunia and sexual dysfunction [27]. It has been shown that depression has negative effects on both CPP and many aspects of sexual function, such as sexual desire, arousal, and orgasm [28]. On the other hand, in another study, although $75.8 \%$ of women described dyspareunia, no negative effect was found on their quality of sexual life [29]. Similarly, in a case-control study in which endometriosis patients and a healthy population were compared, no difference was found between the two groups in terms of sexual dysfunction, although pain scores were higher in patients with endometriosis [30]. In another study involving 300 patients, dyspareunia and decreased sexual desire were observed in patients with deep infiltrative endometriosis and ovarian/peritoneal endometriosis, but sexual dysfunction was not different in these two groups [31].

In the literature, there are some studies investigating pre-and postoperative sexual life quality in patients with endometriosis. Setela et al. reported that postoperative sexual satisfaction increased [32]. In other studies, it was noted that both the frequency of coitus and the quality of sexual life increased after vaginal resection for endometriosis $[33,34]$. It was observed that dyspareunia complaints of the patients regressed at the 6-and 12-month follow-ups after operation, and thus the quality of sexual life increased [35]. In another case-control study, an increase in quality of sexual life was observed in patients with deep infiltrative endometriosis at follow-ups in the 6th and 36th months after the surgery [36]. In a study evaluating patients 2 and 5 years after laparoscopic surgical treatment, they indicated that sexual 
pleasure and coital comfort had increased, and dyspareunia symptoms had regressed compared to their preoperative states [37].

In our study, we found that the dyspareunia scores of the patients decreased and the quality of sexual life improved after surgery. We think that surgical removal of lesions and adhesions lead to improvement in dyspareunia. Regression of pain and psychological relief may have increased the patients' quality of sexual life.

\section{Endometriosis and Quality of Sleep}

Sleep is a physiological requirement for a person to maintain their mental and physical health. Sleep disturbance can lead to serious decreases in daily performance and quality of life, and increases medical, neurological, and psychiatric disorders [38]. Sleep quality may deteriorate with the neurobiological effects of pain. In a study, the pain threshold and sleep quality in patients with endometriosis were examined, and it was found that these patients had significantly poor sleep quality [39]. A recent study found poor sleep quality and more depression in patients with preoperative endometriosis [40]. In a study involving 48 endometriosis patients, sleep disorder was evaluated after medical treatment [41]. In this study, although sleep quality was not evaluated, it was determined that sleep disorder had improved with hormonal therapy after six and 12 months. Most of the studies in the literature evaluated sleep quality in the preoperative period and found deterioration in sleep quality as a result of endometriosis symptoms. In a study by Goksu et al. [11], an increase in sleep quality and a decrease in depression were found in the postoperative period in patients with endometriosis who had stage 4 disease and were operated. Our study is the first in the literature to investigate the quality of sleep in the postoperative period regardless of the stage of endometriosis. The results of our study showed that there was a significant improvement in subjective sleep quality, duration, efficiency, and daytime dysfunction parameters in all patients regardless of stage of endometriosis after surgery.

An individual's circadian typology affects how cognitively and physically active they are throughout the day. People have different circadian types, defined by differences in sleep-wake cycle and biological and behavioral rhythms. Morning types go to bed early and get up early; they perform better in the early morning hours. Evening types stay up late and sleep late and feel more active in the evening. Evening type was found to be associated with short sleep duration, insufficient sleep, and poor sleep quality, and it was stated that insufficient sleep decreased the ability to cope with negative emotions. The relationship between endometriosis and circadian typology has not been adequately examined. In our study, most of the patients who were evening type in the preoperative period changed to intermediate type in the postoperative period. This indicates that their sleep quality likely improved as well.

The strengths of our study were the prospective design and comparison of quality of life, sexual function, and sleep before and after the operation. The main limitation of our study is the relatively small sample size, even though we did enroll a statistically sufficient number of patients. In addition, including longerterm postoperative evaluations may contribute valuable findings to the literature in future studies. 
To the authors' knowledge, our study is the first in the literature to evaluate sleep quality both pre- and postoperatively, as well as the only one to date to examine quality of life, sexual function, and sleep simultaneously and evaluating them in comparison with each other. Our study revealed that there is a potential connection between all conditions and patients diagnosed with endometriosis reported significant improvements in all three areas following surgery, regardless of disease stage. In conclusion, endometriosis is a disease that negatively affects patients' quality of life, sexual function, and sleep, especially due to its causing high levels of pain. In our study, we showed that the pain scores of the patients regressed and their quality of life, sexual life, and sleep were positively affected by definitive surgical treatment. Further studies are needed to clearly understand the effects of endometriosis disease and its treatment on the quality of life, sexual function, and sleep of patients.

\section{Declarations}

Acknowledgements: None

Conflict of Interest: The authors declare that they have no conflict of interest.

Contributions: All authors contributed to the study conception and design. Material preparation, data collection and analysis were performed by Belfin Nur Arici Halici, Meric Kabakci, Gurkan Kiran and Pinar Ozcan. The first draft of the manuscript was written by Belfin Nur Arici Halici and Fatih Aktoz. The article was edited and finalized by Pinar Ozcan. All authors commented on previous versions of the manuscript. All authors read and approved the final manuscript.

Statements and Declarations: The authors declare that there is no conflict of interest.

\section{References}

1. Eskenazi B, Warner ML. Epidemiology of endometriosis. Obstet Gynecol Clin North Am. 1997;24(2):235-58.doi: 10.1016/s0889-8545(05)70302-8.

2. Janssen EB, Rijkers AC, Hoppenbrouwers K, Meuleman C, D'Hooghe TM. Prevalence of endometriosis diagnosed by laparoscopy in adolescents with dysmenorrhea or chronic pelvic pain: a systematic review. Hum Reprod Update. 2013;19(5):570-82.doi: 10.1093/humupd/dmt016.

3. Nnoaham KE, Hummelshoj L, Webster P, d'Hooghe T, de Cicco Nardone F, de Cicco Nardone C, et al. Impact of endometriosis on quality of life and work productivity: a multicenter study across ten countries. Fertil Steril. 2011;96(2):366-73.e8.doi: 10.1016/j.fertnstert.2011.05.090.

4. De Graaff AA, D'Hooghe TM, Dunselman GA, Dirksen CD, Hummelshoj L, Simoens S. The significant effect of endometriosis on physical, mental and social wellbeing: results from an international cross-sectional survey. Hum Reprod. 2013;28(10):2677-85.doi: 10.1093/humrep/det284. 
5. Siedentopf F, Tariverdian N, Rücke M, Kentenich H, Arck PC. Immune status, psychosocial distress and reduced quality of life in infertile patients with endometriosis. Am J Reprod Immunol. 2008;60(5):449-61.doi: 10.1111/j.1600-0897.2008.00644.x.

6. Mclnnes RA. Chronic illness and sexuality. Med J Aust. 2003;179(5):263-6.doi: 10.5694/j.13265377.2003.tb05535.x.

7. Gulec M, Selvi Y, Boysan M, Aydin A, Oral E, Aydin EF. Chronotype effects on general well-being and psychopathology levels in healthy young adults. Biological Rhythm Research. 2013;44(3):457-68.doi: 10.1080/09291016.2012.704795.

8. Loprinzi PD, Joyner C. Meeting Sleep Guidelines Is Associated With Better Health-Related Quality of Life and Reduced Premature All-Cause Mortality Risk. Am J Health Promot. 2018;32(1):68-71.doi: $10.1177 / 0890117116687459$.

9. Buysse DJ, Reynolds CF, 3rd, Monk TH, Berman SR, Kupfer DJ. The Pittsburgh Sleep Quality Index: a new instrument for psychiatric practice and research. Psychiatry Res. 1989;28(2):193-213.doi:

10.1016/0165-1781(89)90047-4.

10. Nezhat C, Vang N, Tanaka PP, Nezhat C. Optimal Management of Endometriosis and Pain. Obstet Gynecol. 2019;134(4):834-9.doi: 10.1097/aog.0000000000003461.

11. Goksu M, Kadirogullari P, Seckin KD. Evaluation of depression and sleep disorders in the preoperative and postoperative period in stage 4 endometriosis patients. Eur J Obstet Gynecol Reprod Biol. 2021;264:254-8.doi: 10.1016/j.ejogrb.2021.07.037.

12. Ball E, Khan KS. Recent advances in understanding and managing chronic pelvic pain in women with special consideration to endometriosis. F1000Res. 2020;9.doi: 10.12688/f1000research.20750.1.

13. Milingos S, Protopapas A, Kallipolitis G, Drakakis P, Loutradis D, Liapi A, et al. Endometriosis in patients with chronic pelvic pain: is staging predictive of the efficacy of laparoscopic surgery in pain relief? Gynecol Obstet Invest. 2006;62(1):48-54.doi: 10.1159/000092023.

14. Fourquet J, Gao X, Zavala D, Orengo JC, Abac S, Ruiz A, et al. Patients' report on how endometriosis affects health, work, and daily life. Fertil Steril. 2010;93(7):2424-8.doi:

10.1016/j.fertnstert.2009.09.017.

15. Vitale SG, La Rosa VL, Rapisarda AMC, Laganà AS. Impact of endometriosis on quality of life and psychological well-being. J Psychosom Obstet Gynaecol. 2017;38(4):317-9.doi:

10.1080/0167482x.2016.1244185.

16. La Rosa VL, De Franciscis P, Barra F, Schiattarella A, Török P, Shah M, et al. Quality of life in women with endometriosis: a narrative overview. Minerva Med. 2020;111(1):68-78.doi: 10.23736/s00264806.19.06298-0. 
17. Márki G, Bokor A, Rigó J, Rigó A. Physical pain and emotion regulation as the main predictive factors of health-related quality of life in women living with endometriosis. Hum Reprod. 2017;32(7):1432-8.doi: 10.1093/humrep/dex091.

18. Rush G, Misajon R. Examining subjective wellbeing and health-related quality of life in women with endometriosis. Health Care Women Int. 2018;39(3):303-21.doi: 10.1080/07399332.2017.1397671.

19. Ayan M, Taş U, Söğüt E, Arıcı S, Karaman S, Esen M, et al. [Comparing effıciencies of diclofenac sodium and paracetamol in patients with primary dysmenorrhea pain by using Visual Analog Scale]. Agri. 2013;25(2):78-82.doi: 10.5505/agri.2013.42103.

20. Parra RS, Feitosa MR, Camargo HP, Valério FP, Zanardi JVC, Rocha J, et al. The impact of laparoscopic surgery on the symptoms and wellbeing of patients with deep infiltrating endometriosis and bowel involvement. J Psychosom Obstet Gynaecol. 2021;42(1):75-80.doi:

10.1080/0167482x.2020.1773785.

21. Bastu E, Celik HG, Kocyigit Y, Yozgatli D, Yasa C, Ozaltin S, et al. Improvement in quality of life and pain scores after laparoscopic management of deep endometriosis: a retrospective cohort study. Arch Gynecol Obstet. 2020;302(1):165-72.doi: 10.1007/s00404-020-05583-6.

22. Rindos NB, Fulcher IR, Donnellan NM. Pain and Quality of Life after Laparoscopic Excision of Endometriosis. J Minim Invasive Gynecol. 2020;27(7):1610-7.e1.doi: 10.1016/j.jmig.2020.03.013.

23. Abbott J, Hawe J, Hunter D, Holmes M, Finn P, Garry R. Laparoscopic excision of endometriosis: a randomized, placebo-controlled trial. Fertil Steril. 2004;82(4):878-84.doi:

10.1016/j.fertnstert.2004.03.046.

24. Pluchino N, Wenger JM, Petignat $\mathrm{P}, \mathrm{Tal} \mathrm{R}$, Bolmont M, Taylor HS, et al. Sexual function in endometriosis patients and their partners: effect of the disease and consequences of treatment. Hum Reprod Update. 2016;22(6):762-74.doi: 10.1093/humupd/dmw031.

25. Yong PJ. Deep Dyspareunia in Endometriosis: A Proposed Framework Based on Pain Mechanisms and Genito-Pelvic Pain Penetration Disorder. Sex Med Rev. 2017;5(4):495-507.doi: 10.1016/j.sxmr.2017.06.005.

26. Melis I, Litta P, Nappi L, Agus M, Melis GB, Angioni S. Sexual Function in Women with Deep Endometriosis: Correlation with Quality of Life, Intensity of Pain, Depression, Anxiety, and Body Image. International Journal of Sexual Health. 2015;27(2):175-85.doi: 10.1080/19317611.2014.952394.

27. Shum LK, Bedaiwy MA, Allaire C, Williams C, Noga H, Albert A, et al. Deep Dyspareunia and Sexual Quality of Life in Women With Endometriosis. Sex Med. 2018;6(3):224-33.doi: 10.1016/j.esxm.2018.04.006. 
28. Cyranowski JM, Frank E, Cherry C, Houck P, Kupfer DJ. Prospective assessment of sexual function in women treated for recurrent major depression. J Psychiatr Res. 2004;38(3):267-73.doi:

10.1016/j.jpsychires.2003.08.003.

29. Kiykac Altinbas S, Bayoglu Tekin Y, Dilbaz B, Dilbaz S. Evaluation of quality of life in fertile Turkish women with severe endometriosis. J Obstet Gynaecol. 2015;35(1):49-52.doi:

10.3109/01443615.2014.930110.

30. Evangelista A, Dantas T, Zendron C, Soares T, Vaz G, Oliveira MA. Sexual function in patients with deep infiltrating endometriosis. J Sex Med. 2014;11(1):140-5.doi: 10.1111/jsm.12349.

31. Vercellini P, Somigliana E, Buggio L, Barbara G, Frattaruolo MP, Fedele L. "I can't get no satisfaction": deep dyspareunia and sexual functioning in women with rectovaginal endometriosis. Fertil Steril. 2012;98(6):1503-11.e1.doi: 10.1016/j.fertnstert.2012.07.1129.

32. Setälä M, Härkki P, Matomäki J, Mäkinen J, Kössi J. Sexual functioning, quality of life and pelvic pain 12 months after endometriosis surgery including vaginal resection. Acta Obstet Gynecol Scand. 2012;91(6):692-8.doi: 10.1111/j.1600-0412.2012.01394.x.

33. Kristensen J, Kjer JJ. Laparoscopic laser resection of rectovaginal pouch and rectovaginal septum endometriosis: the impact on pelvic pain and quality of life. Acta Obstet Gynecol Scand. 2007;86(12):1467-71.doi: 10.1080/00016340701645006.

34. Anaf V, Simon P, El Nakadi I, Simonart T, Noel J, Buxant F. Impact of surgical resection of rectovaginal pouch of douglas endometriotic nodules on pelvic pain and some elements of patients' sex life. J Am Assoc Gynecol Laparosc. 2001;8(1):55-60.doi: 10.1016/s1074-3804(05)60549-x.

35. Ferrero S, Abbamonte LH, Giordano M, Ragni N, Remorgida V. Deep dyspareunia and sex life after laparoscopic excision of endometriosis. Hum Reprod. 2007;22(4):1142-8.doi: 10.1093/humrep/del465.

36. Martínez-Zamora MA, Coloma JL, Gracia M, Rius M, Castelo-Branco C, Carmona F. Long-term Follow-up of Sexual Quality of Life after Laparoscopic Surgery in Patients with Deep Infiltrating Endometriosis. J Minim Invasive Gynecol. 2021.doi: 10.1016/j.jmig.2021.04.023.

37. Abbott JA, Hawe J, Clayton RD, Garry R. The effects and effectiveness of laparoscopic excision of endometriosis: a prospective study with 2-5 year follow-up. Hum Reprod. 2003;18(9):1922-7.doi: 10.1093/humrep/deg275.

38. Guallar-Castillón P, Bayán-Bravo A, León-Muñoz LM, Balboa-Castillo T, López-García E, GutierrezFisac JL, et al. The association of major patterns of physical activity, sedentary behavior and sleep with health-related quality of life: a cohort study. Prev Med. 2014;67:248-54.doi:

10.1016/j.ypmed.2014.08.015. 
39. Nunes FR, Ferreira JM, Bahamondes L. Pain threshold and sleep quality in women with endometriosis. Eur J Pain. 2015;19(1):15-20.doi: 10.1002/ejp.514.

40. Arion K, Orr NL, Noga H, Allaire C, Williams C, Bedaiwy MA, et al. A Quantitative Analysis of Sleep Quality in Women with Endometriosis. J Womens Health (Larchmt). 2020;29(9):1209-15.doi: 10.1089/jwh.2019.8008.

41. Bergqvist A, Theorell T. Changes in quality of life after hormonal treatment of endometriosis. Acta Obstet Gynecol Scand. 2001;80(7):628-37. 